Article

\title{
Practical Asymmetric Synthesis of Sitagliptin Phosphate Monohydrate
}

\author{
Haoling Gao ${ }^{1,2}$, Jiangang Yu ${ }^{1}$, Chengsheng Ge ${ }^{1,2, *}$ and Qun Jiang ${ }^{2}$ \\ 1 College of Chemistry and Materials Engineering, Quzhou University, Quzhou 324000, China; \\ xjguo631@sina.com (H.G.); jgyu@qzu.zj.cn (J.Y.) \\ 2 College of Chemical Engineering, Zhejiang University of Technology, Hangzhou 310014, China; \\ jiangqun1991@163.com \\ * Correspondence: gechengsheng@qzu.zj.cn; Tel.: +86-570-8012508
}

Received: 3 June 2018; Accepted: 12 June 2018; Published: 13 June 2018

check for updates

\begin{abstract}
Optically pure sitagliptin phosphate monohydrate is efficiently and practically synthesized through a chiral hemiacetal as the key intermediate in 54\% overall yield starting from (E)-4-(2,4,5-trifluorophenyl)but-2-enal and N-boc-protected hydroxylamine. The chiral hemiacetal fragment is constructed by a tandem aza-Michael/hemiacetal reaction catalyzed by an organocatalyst and the influence of acidity of Brønsted acid on tandem aza-Michael/hemiacetal reaction is researched in detail.
\end{abstract}

Keywords: sitagliptin phosphate monohydrate; aza-Michael/hemiacetal reaction; asymmetric synthesis

\section{Introduction}

Since its launch in 2006 by Merck, sitagliptin phosphate monohydrate, the first DPP-4 inhibitor (dipeptidyl peptidase 4), has been one of the best-selling orally active and safe agents for the treatment of T2DM (type 2 diabetes mellitus) [1-3]. Considerable efforts have been made on searching for a short, environmentally friendly, and economic asymmetric synthetic route toward sitagliptin phosphate monohydrate including Merck's three-generation process. In this process, the key structure of sitagliptin was installed via rare-metal catalyzed asymmetric hydrogenation, biocatalytic asymmetric reduction or transaminase-catalyzed asymmetric synthesis [4,5]. Generally, most of synthetic routes including the latest reported methods focus on constructing key chiral fragment by employing diverse metal catalysts [6-8], chiral auxiliary [9-12] or chiral starting materials [13,14].

In the last decades, a variety of chiral amino acids can be made from hemiacetals through catalytic tandem aza-Michael/hemiacetal reaction, using organocatalyst in high yield and enantioselectivity [15-19]. As part of our efforts to develop efficient and practical access to stigliptin phosphate monohydrate, we herein report an efficient synthetic route to make sitagliptin phosphate monohydrate by means of chiral hemiacetal as the key intermediate.

\section{Results and Discussion}

Initially, the model aza-Michael addition reaction that we studied, between (E)-4-phenylbut-2-enal $1 \mathrm{a}$ and $\mathrm{N}$-Boc-protected hydroxylamine or $\mathrm{N}$-Cbz-protected hydroxylamine catalyzed by organocatalyst I or II in chloroform at $0{ }^{\circ} \mathrm{C}$ did not take place and obtained the starting materials were only. However, when the mixture of $20 \mathrm{~mol} \%$ benzoic acid ( $\mathrm{pKa}=4.19$ ) [20] and $20 \mathrm{~mol} \%$ piperidine were used as a catalyst, the tandem aza-Michael/hemiacetal reaction took place to give an unexpected macro-cyclic compound 3 in 91\% isolated yield (Scheme 1). In contrast, macro-cyclic compound 3 and $N$-Boc hemiacetal 4 a was isolated in 11\% and 71\% yields, respectively, by using 20 mol \% chiral secondary amine II together with $20 \mathrm{~mol} \%$ benzoic acid as catalyst, and the enantiomeric excess 
(ee) of $N$-Boc hemiacetal 4a up to $89 \%$ (Scheme 2). Obviously, the tandem aza-Michael/hemiacetal reaction adduct is different from the outcome of tandem aza-Michael/hemiacetal reaction between cinnamaldehyde and N-Boc-protected hydroxylamine in the literature with regard to distinguishable products [17]. We suppose that formyl group of aza-Michael addition adduct was activated by Brønsted acid (benzoic acid) to facilitate intramolecular hemiacetal formation to complete the tandem aza-Michael/hemiacetal reaction. We assume the acidity of Brønsted acid had great effect on the outcome of tandem aza-Michael/hemiacetal reaction.

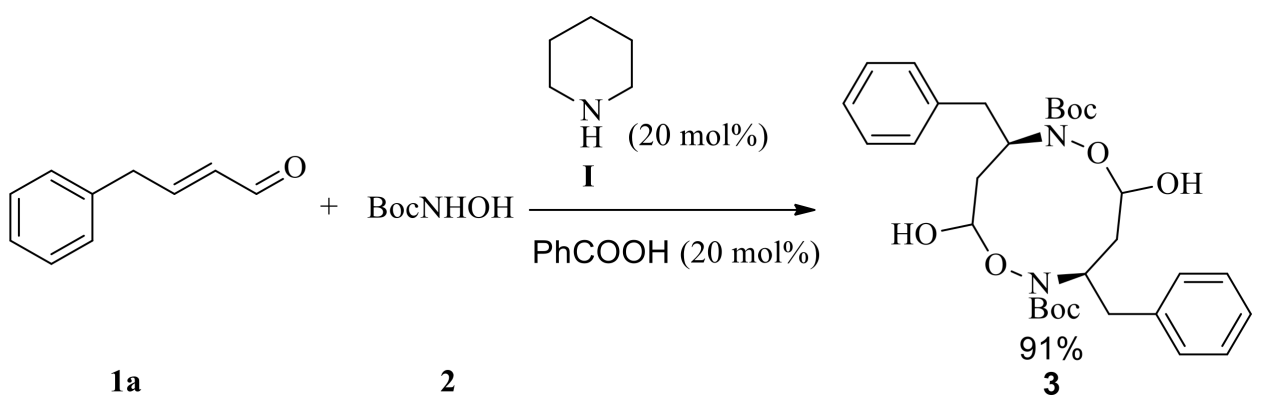

Scheme 1. Tandem aza-Michael/hemiacetal reaction between (E)-4-phenyl)but-2-enal 1a and $\mathrm{N}$-Boc-protected hydroxylamine (2) catalyzed by piperidine (I) and benzoic acid to give 3,8-dibenzyl-5,10-dihydroxy-1,6,2,7-dioxadiazecane-2,7-dicarboxylate (3).

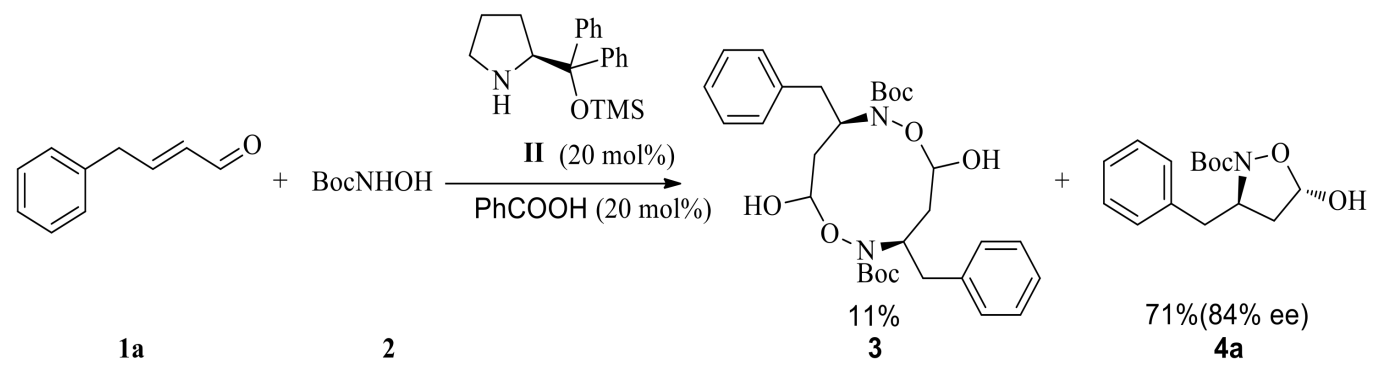

Scheme 2. Tandem aza-Michael/hemiacetal reaction between (E)-4-phenyl)but-2-enal 1a and $N$-Boc-protected hydroxylamine (2) catalyzed by catalyst II and benzoic acid to give 3,8-dibenzyl-5,10-dihydroxy-1,6,2,7-dioxadiazecane-2,7-dicarboxylate (3) and (3R,5S)-tert-butyl 3-benzyl-5-hydroxyisoxazolidine-2-carboxylate (4a).

The evaluation on the influence of different Brønsted acid was shown in Table 1. When the stronger Brønsted acids, such as phosphoric acid $(\mathrm{pKa}=2.12)$, trifluoroacetic acid $(\mathrm{pKa}=0.23)$ and $p$-toluenesulfonic acid ( $\mathrm{pKa}=-2.8$ ), were used as additives, macro-cyclic compound 3 could not be found, however, N-Boc hemiacetal $\mathbf{4 a}$ and double Boc derivative 5 were isolated. Meanwhile, the enantioselectivities were low at the presence of stronger Brønsted acids. Fortunately, hemiacetal 4a was isolated as single product in $84 \%$ yield with $92 \%$ ee using 20 mol \% $p$-nitrobenzoic acid (PNBA, $\mathrm{pKa}=3.47)$ as an additive, together with $20 \mathrm{~mol} \%(S)$-diphenylprolinol-TMS II. It seems that Brønsted acid can effectively activate the formyl group of the aza-Michael addition adduct to facilitate the sequence, and acidity of Brønsted acid can mediate the outcome of tandem aza-Michael/hemiacetal reaction. In other solvents, such as diethyl ether, methanol, toluene, and dichloromethane, tandem aza-Michael/hemiacetal reaction catalyzed by (S)-diphenylprolinol-TMS II together with $p$-nitrobenzoic acid worked smoothly (Table 1, entries 5-8). Dichloromethane was the best solvent, as $90 \%$ yield and $92 \%$ ee can be obtained even when the loading amount of (S)-diphenylprolinol-TMS II and additive was reduced to $10 \mathrm{~mol} \%$ (entry 9). Catalyst III and IV were also tested (entries 10-11), and it was found that catalyst III, containing a 3,5-ditrifluoromethylphenyl group, gave 45\% ee, while catalyst IV afforded product in low yield with poor enantioselectivity. 
Table 1. Tandem aza-Michael/hemiacetal reaction between (E)-4-phenylbut-2-enal 1a and $\mathrm{N}$-Boc-hydroxylamine at the presence of secondary amine and Brønsted acid additives a .

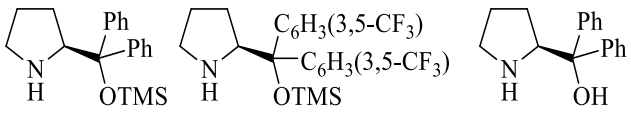

III

IV

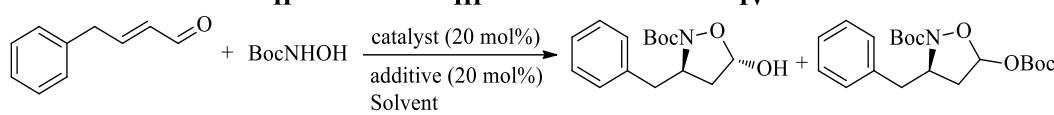

1a 2

\begin{tabular}{|c|c|c|c|c|}
\hline Entry & Cat. & Solvent & Additive & Product $\left(\%\right.$ Yield $^{b}, \%$ ee $\left.^{c}\right)$ \\
\hline 1 & II & $\mathrm{CHCl}_{3}$ & $\mathrm{H}_{3} \mathrm{PO}_{4}$ & $\mathbf{4 a}(21,43)+\mathbf{5}(35,43)$ \\
\hline 2 & II & $\mathrm{CHCl}_{3}$ & TFA & $4 \mathbf{a}(18,41)+5(42,42)$ \\
\hline 3 & II & $\mathrm{CHCl}_{3}$ & $p-\mathrm{TsOH}$ & $\mathbf{4 a}(8,44)+5(65,43)$ \\
\hline 4 & II & $\mathrm{CHCl}_{3}$ & $p-\mathrm{NO}_{2} \mathrm{C}_{6} \mathrm{H}_{4} \mathrm{COOH}$ & $4 \mathbf{a}(84,92)$ \\
\hline 5 & II & $\mathrm{Et}_{2} \mathrm{O}$ & $p-\mathrm{NO}_{2} \mathrm{C}_{6} \mathrm{H}_{4} \mathrm{COOH}$ & $4 a(78,86)$ \\
\hline 6 & II & $\mathrm{MeOH}$ & $p-\mathrm{NO}_{2} \mathrm{C}_{6} \mathrm{H}_{4} \mathrm{COOH}$ & $4 \mathbf{a}(72,76)$ \\
\hline 7 & II & Toluene & $p-\mathrm{NO}_{2} \mathrm{C}_{6} \mathrm{H}_{4} \mathrm{COOH}$ & $4 \mathbf{a}(77,80)$ \\
\hline 8 & II & DCM & $p-\mathrm{NO}_{2} \mathrm{C}_{6} \mathrm{H}_{4} \mathrm{COOH}$ & $\mathbf{4 a}(90,92)$ \\
\hline 9 & II & DCM & $p-\mathrm{NO}_{2} \mathrm{C}_{6} \mathrm{H}_{4} \mathrm{COOH}$ & $4 \mathbf{a}(90,92){ }^{d}$ \\
\hline 10 & III & DCM & $p-\mathrm{NO}_{2} \mathrm{C}_{6} \mathrm{H}_{4} \mathrm{COOH}$ & $\mathbf{4 a}(79,45)$ \\
\hline 11 & IV & DCM & $p-\mathrm{NO}_{2} \mathrm{C}_{6} \mathrm{H}_{4} \mathrm{COOH}$ & $4 \mathbf{a}(30,42)$ \\
\hline
\end{tabular}

a All reactions were carried out using (E)-4-phenylbut-2-enal 1a (146 mg, $1 \mathrm{mmol}$ ), $N$-Boc-hydroxylamine $2(199 \mathrm{mg}, 1.5 \mathrm{mmol})$ and catalyst $(0.2 \mathrm{mmol}, 20 \mathrm{~mol} \%)$ in solvent $(5 \mathrm{~mL})$ in the presence of the indicated Brønsted acid additives $(20 \mathrm{~mol} \%)$ at $0{ }^{\circ} \mathrm{C}$ for $8 \mathrm{~h}$. ${ }^{\mathrm{b}}$ Isolated yield after silica-gel column chromatography. ${ }^{c}$ Determined by a chiral HPLC analysis of the isolated products. ${ }^{\mathrm{d}} 10 \mathrm{~mol} \%$ of (S)-diphenylprolinol-TMS II and $10 \mathrm{~mol} \%$ of p-nitrobenzoic acid were applied to the reaction and the reaction was run at $0{ }^{\circ} \mathrm{C}$ for $15 \mathrm{~h}$.

(E)-4-(2,4,5-Trifluorophenyl)but-2-enal $\mathbf{1 b}$ was obtained via either the cross-metathesis reaction between 1-allyl-2,4,5-trifluorobenzene and crotonaldehyde or the coupling reaction between 1-bromo-2,4,5-trifluorobenzene and (E)-4-bromo-1,1-dimethoxybut-2-ene [21]. Tandem aza-Michael/ hemiacetal reaction between (E)-4-(2,4,5-trifluorophenyl)but-2-enal $\mathbf{1 b}$ and $N$-Boc-hydroxylamine catalyzed by $10 \mathrm{~mol} \%$ (S)-diphenylprolinol-TMS II and $10 \mathrm{~mol} \%$ p-nitrobenzoic acid gave $(3 R, 5 S)-N$-Boc-5-hydroxy-3-(2,4,5-trifluorobenzyl)isoxazolidine $4 \mathrm{~b}$ in $85 \%$ yield and $93 \%$ ee (Scheme 3 ). Decreasing the reaction temperature to $-20{ }^{\circ} \mathrm{C}$ improved the enantioselectivty. The ee was up to $96 \%$ with an extended reaction time ( $15 \mathrm{~h})$, and yield was up to $85 \%$.<smiles>O=C/C=C/Cc1cc(F)c(F)cc1F</smiles>

$1 \mathrm{~b}$<smiles>O[C@H]1CC(Cc2cc(F)c(F)cc2F)[C@H](O)ON1</smiles>

$4 \mathrm{~b}$

Scheme 3. Tandem aza-Michael/hemiacetal reaction between (E)-4-(2,4,5-trifluorophenyl)but-2-enal $\mathbf{1 b}$ and N-Boc-protected hydroxylamine (2) to give (3R,5S)-tert-butyl 5-hydroxy-3-(2,4,5-trifluorobenzyl) isoxazolidine-2-carboxylate $(4 \mathbf{b})$.

We next converted this key chiral unit to chiral $\beta$-amino acid. Oxidation/reductive cleavage of $\mathrm{N}-\mathrm{O}$ bond sequence are a common methodology for this conversion. Chiral $(R)-N$-Boc- $\beta$-benzyl$\beta$-amino acid 7a was obtained via $\mathrm{NaClO}_{2} / \mathrm{H}_{2} \mathrm{O}_{2} / \mathrm{NaH}_{2} \mathrm{PO}_{4}$ oxidation/hydrogenation, starting from hemiacetal $4 \mathrm{a}$ in $82 \%$ yield, without the loss of enantiomeric purity (Scheme 4 ). However, 
$\mathrm{NaClO}_{2} / \mathrm{H}_{2} \mathrm{O}_{2} / \mathrm{NaH}_{2} \mathrm{PO}_{4}$ oxidation/hydrogenation sequence starting from hemiacetal $4 \mathbf{b}$ gave (R)- $N$-Boc- $\beta$-(2,4,5-trifluorobenzyl)- $\beta$-amino acid $7 \mathbf{b}$ in poor yield (32\% in total).

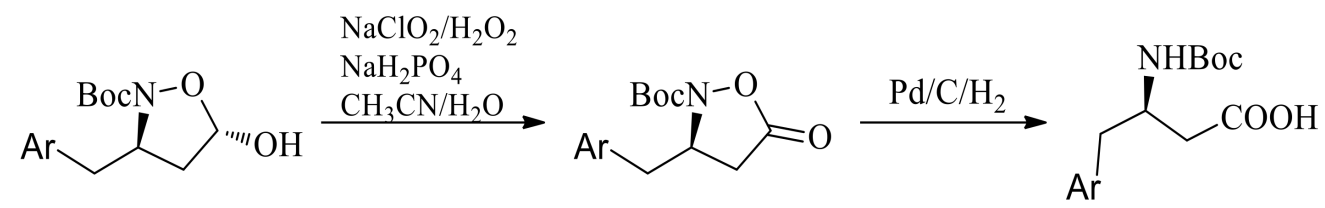
4a: $\mathrm{Ar}=\mathrm{Ph}$
6a: $\mathrm{Ar}=\mathrm{Ph}$
7a: $\mathrm{Ar}=\mathrm{Ph}, 81 \%$ total yield
4b: $\mathrm{Ar}=2,4,5$-trifluoroPh
6b: $\mathrm{Ar}=2,4,5$-trifluoroPh
$7 \mathbf{b}: \mathrm{Ar}=2,4,5$-trifluoroPh, $32 \%$ total yield

Scheme 4. Oxidation/reductive cleavage of N-O bond consequence.

We then turned our attention to obtain chiral $\beta$-amino acid via reductive cleavage of the $\mathrm{N}-\mathrm{O}$ bond/oxidation sequence (Scheme 5). $\mathrm{N}-\mathrm{O}$ bond reductive cleavage using $\mathrm{Mo}(\mathrm{CO})_{6}$ as the reducing agent, followed by oxidation, gave the $\beta$-amino acid $7 \mathbf{a}$ and $7 \mathbf{b}$ in $83 \%$ and $82 \%$ yields, respectively. Moreover, reduction with $\mathrm{NaBH}_{4}$, followed by N-O bond cleavage via hydrogenation with Raney $\mathrm{Ni}$ gave the $\beta$-amino alcohol $7 \mathrm{a}$ and $7 \mathrm{~b}$ in $83 \%$ and $82 \%$ yields respectively. At a pressure of 90 atm of hydrogen and $\mathrm{Pd} / \mathrm{C}$, the $\beta$-amino alcohol $\mathbf{9 a}$ and $\mathbf{9 b}$ were obtained in $99 \%$ yield. Further oxidation of $\beta$-amino alcohol $9 \mathbf{a}$ and $\mathbf{9 b}$ with $\mathrm{NaClO} / \mathrm{TEMPO}$ gave the $\beta$-amino acid $7 \mathbf{a}$ and $7 \mathbf{b}$ in $92 \%$ and $91 \%$ yield respectively. Compared with $\mathrm{N}-\mathrm{O}$ bond reductive cleavage using expensive $\mathrm{Mo}(\mathrm{CO})_{6}$ or $\mathrm{NaBH}_{4} /$ hydrogenation sequence, $\mathrm{N}-\mathrm{O}$ bond reductive cleavage using hydrogenation have industrial potential.

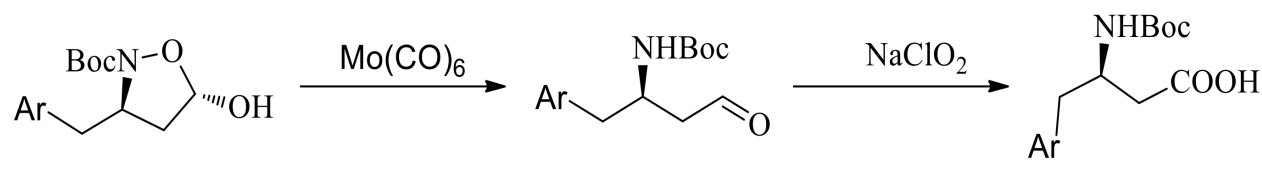

4a: $\mathrm{Ar}=\mathrm{Ph}$

4b: $\mathrm{Ar}=2,4,5$-trifluoroPh 8a: $\mathrm{Ar}=\mathrm{Ph}$

8b: $\mathrm{Ar}=2,4,5$-trifluoroPh 7a: $\mathrm{Ar}=\mathrm{Ph}, 83 \%$ total yield

7b: $\mathrm{Ar}=(2,4,5$-trifluoro $) \mathrm{Ph}, 82 \%$ total yield

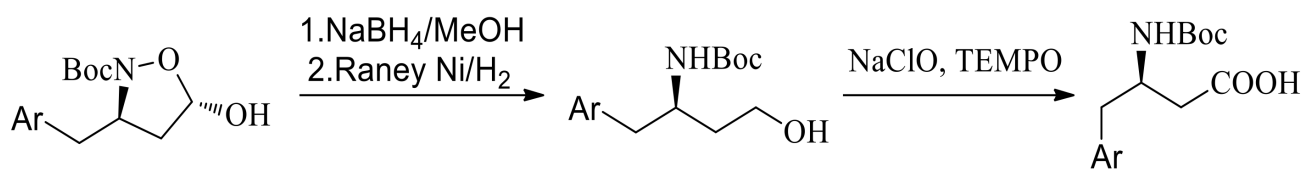

4a: $\mathrm{Ar}=\mathrm{Ph}$

4b: $\mathrm{Ar}=2,4,5$-trifluoroPh 9a: $\mathrm{Ar}=\mathrm{Ph}, 83 \%$ yield 7a: $\mathrm{Ar}=\mathrm{Ph}, 89 \%$ yield

$7 \mathbf{b}: \mathrm{Ar}=2,4,5$-trifluoro $\mathrm{Ph}, 91 \%$ yield

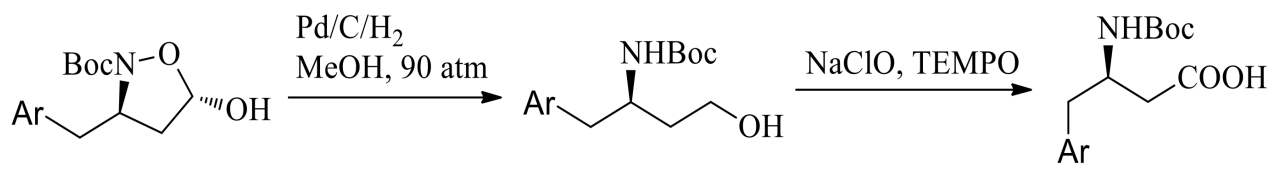

4a: $\mathrm{Ar}=\mathrm{Ph}$

9a: $\mathrm{Ar}=\mathrm{Ph}, 99 \%$ yield

4b: $\mathrm{Ar}=2,4,5$-trifluoroPh 9b: $\mathrm{Ar}=2,4,5$-trifluoroPh, 99\% yield $7 \mathbf{b}$ : $\mathrm{Ar}=2,4,5$-trifluoroPh, $91 \%$ yield

Scheme 5. Reductive cleavage of N-O bond/oxidation sequence.

(R)-N-Boc- $\beta$-(2,4,5-trifluorobenzyl)- $\beta$-amino acid $7 \mathbf{b}$ was synthesized starting from $(E)-4-(2,4,5-$ trifluorophenyl)but-2-enal in $76 \%$ total yield in a three-step procedure (Scheme 6). (R)-tert-butyl 4-oxo-4-(3-(trifluoromethyl)-5,6-dihydro-[1,2,4]triazolo[4,3-a]pyrazin-7(8H)-yl)-1-(2,4,5-tri-fluorophenyl) butan-2-ylcarbamate 11 was obtained in 91\% yield via the coupling reaction between triazole (10) and (R)-N-Boc- $\beta$-(2,4,5-trifluorobenzyl)- $\beta$-amino acid $7 \mathbf{b}$, using HOBt-EDCI as the coupling agent. Finally, 
$>99.9 \%$ ee of sitagliptin phosphate monohydrate was provided in $78 \%$ yield following the known literature procedure (as shown in Scheme 6) [6].
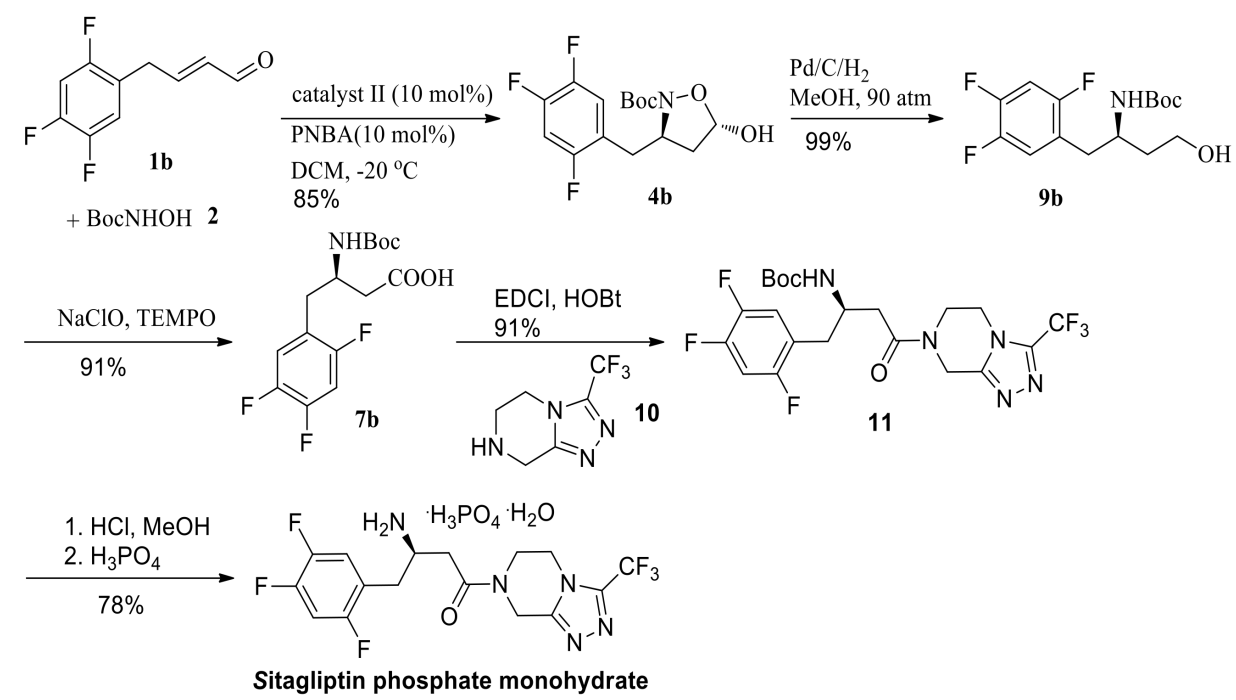

Scheme 6. Synthesis of sitagliptin phosphate monohydrate.

\section{Materials and Methods}

\subsection{General}

Unless otherwise noted, all commercial reagents were used as purchased from Aladdin and Tansoole (Chinese chemical reagents supplier). All the reactions were monitored by thin-layer chromatography (TLC) that was performed on silica gel plates GF254. Visualization was achieved under a UV lamp, and by developing the plates with Potassium Permanganate in water. Flash chromatography was performed using silica gel (200-300 mesh) with solvents indicated in the text. NMR spectra were registered in a Bruker Advance 400 Ultrashield spectrometer at room temperature, operating at 400 $\mathrm{MHz}\left({ }^{1} \mathrm{H}\right)$ and $100 \mathrm{MHz}\left({ }^{13} \mathrm{C}\right)$. The experiments of high performance liquid chromatography (HPLC) was performed on a Shimadzu chromatograph (Essentia LC-16, Shimadzu Corp., Kyoto, Japan) by using Chiralcel columns as illustrated in supporting information.

\subsection{Synthesis of (E)-4-Phenylbut-2-enal (1a)}

A solution of allylbenzene $(591 \mathrm{mg}, 5 \mathrm{mmol})$ and crotonaldehyde $(700 \mathrm{mg}, 10 \mathrm{mmol})$ together with $0.1 \mathrm{~mol} \%$ Grubbs-II catalyst $(4.2 \mathrm{mg}, 0.005 \mathrm{mmol})$ in dichloromethane $(20 \mathrm{~mL})$ was refluxed for $12 \mathrm{~h}$, and then cooled to $0{ }^{\circ} \mathrm{C}$. The mixture solution was concentrated under reduced pressure and purified over silica gel column chromatography (PE/EtOAc, 20:1-10:1) to give 1a as a liquid (650 mg, $89 \%) .{ }^{1} \mathrm{H}-\mathrm{NMR}\left(400 \mathrm{MHz}, \mathrm{CDCl}_{3}\right) \delta 9.52(\mathrm{~d}, J=8.2 \mathrm{~Hz}, 1 \mathrm{H}), 7.34-7.31(\mathrm{~m}, 2 \mathrm{H}), 7.27-7.23(\mathrm{~m}, 1 \mathrm{H})$, $7.17(\mathrm{~d}, J=8.5 \mathrm{~Hz}, 2 \mathrm{H}), 6.99-6.91(\mathrm{~m}, 1 \mathrm{H}), 6.10(\mathrm{dd}, J=8.1 \mathrm{~Hz}$ and $8 \mathrm{~Hz}, 1 \mathrm{H}), 3.64(\mathrm{~d}, J=4.2 \mathrm{~Hz}, 2 \mathrm{H})$ (Supplementary Material Figure S1).

\subsection{Synthesis of (E)-4-(2,4,5-Trifluorophenyl)but-2-enal (1b)}

A solution of 1-allyl-2,4,5-trifluorobenzene $(860 \mathrm{mg}, 5 \mathrm{mmol})$ and crotonaldehyde (700 $\mathrm{mg}$, $10 \mathrm{mmol})$, together with $0.1 \mathrm{~mol} \%$ Grubbs-II catalyst $(4.2 \mathrm{mg}, 0.005 \mathrm{mmol})$ in dichloromethane $(20 \mathrm{~mL})$, was refluxed for $12 \mathrm{~h}$, and then it was cooled to $0{ }^{\circ} \mathrm{C}$. The mixture solution was concentrated under reduced pressure and purified over silica gel column chromatography (PE/EtOAc, 20:1-10:1) to give $1 \mathbf{b}$ as a liquid $(830 \mathrm{mg}, 83 \%) .{ }^{1} \mathrm{H}-\mathrm{NMR}\left(400 \mathrm{MHz}, \mathrm{CDCl}_{3}\right) \delta 9.52(\mathrm{~d}, J=8.6 \mathrm{~Hz}, 1 \mathrm{H}), 7.02-6.83(\mathrm{~m}$, $3 \mathrm{H}), 6.08-6.02(\mathrm{~m}, 1 \mathrm{H}), 3.60(\mathrm{~d}, J=4.4 \mathrm{~Hz}, 2 \mathrm{H})$ (Supplementary Material Figure S2). 


\subsection{Synthesis of Di-tert-butyl 3,8-Dibenzyl-5,10-dihydroxy-1,6,2,7-dioxadiazecane-2,7-dicarboxylate (3)}

(E)-4-phenylbut-2-enal 1a (146 mg, $1 \mathrm{mmol})$ was dissolved in dichloromethane $(5 \mathrm{~mL})$, and then $\mathrm{N}$-Boc-hydroxylamine 2 (199 mg, $1.5 \mathrm{mmol})$, piperidine $(17 \mathrm{mg}, 0.2 \mathrm{mmol})$, and benzoic acid (24 mg, $0.2 \mathrm{mmol}$ ) were added to the resulting solution at $0{ }^{\circ} \mathrm{C}$. The reaction mixture was stirred for $8 \mathrm{~h}$ at $0{ }^{\circ} \mathrm{C}$. The mixture solution was diluted with dichloromethane $(20 \mathrm{~mL})$ and washed with saturated sodium bicarbonate aqueous solution $(5 \mathrm{~mL})$ and brine $(5 \mathrm{~mL})$. The resulting organic phase was dried over $\mathrm{Na}_{2} \mathrm{SO}_{4}$, filtered and concentrated under reduced pressure. The crude product was purified over silica gel column chromatography (PE/EtOAc, 10:1-4:1) to give 3 as a white solid (254 mg, 91\%). mp: 123-124 ${ }^{\circ} \mathrm{C} ;{ }^{1} \mathrm{H}-\mathrm{NMR}\left(400 \mathrm{MHz}, \mathrm{CDCl}_{3}\right) \delta$ 7.32-7.22 (m, 10H), 5.73-5.69 (m, 2H), 4.66-4.59 (m, 1H), 4.31-4.24 (m, 1H), $3.43(\mathrm{~s}, 1 \mathrm{H}), 3.21-3.17(\mathrm{~m}, 2 \mathrm{H}), 3.11(\mathrm{dd}, J=4.2 \mathrm{~Hz}, 12 \mathrm{~Hz}, 1 \mathrm{H}), 2.94(\mathrm{dd}, J=4.2 \mathrm{~Hz}$, $12 \mathrm{~Hz}, 1 \mathrm{H}), 2.74(\mathrm{dd}, J=4.1 \mathrm{~Hz}, 12 \mathrm{~Hz}, 1 \mathrm{H}), 2.58-2.51(\mathrm{~m}, 1 \mathrm{H}), 2.24(\mathrm{dd}, J=4.1 \mathrm{~Hz}, 12 \mathrm{~Hz}, 1 \mathrm{H}), 2.16-2.09$ $(\mathrm{m}, 1 \mathrm{H}), 2.06-1.99(\mathrm{~m}, 1 \mathrm{H}), 1.53(\mathrm{~s}, 9 \mathrm{H}), 1.51(\mathrm{~s}, 9 \mathrm{H})$ (Supplementary Material Figure S3); ${ }^{13} \mathrm{C}-\mathrm{NMR}$ $\left(100 \mathrm{MHz}_{,} \mathrm{CDCl}_{3}\right) \delta$ 155.0, 154.0, 137.0, 136.8, 129.2, 129.2, 128.5, 128.5, 126.7, 126.6, 83.5, 82.5, 82.5, 81.9, 81.5, 79.8, 41.8, 41.0, 39.2, 38.8, 28.3, 28.2 (Supplementary Material Figure S4); HR-MS $\left(\mathrm{C}_{30} \mathrm{H}_{42} \mathrm{~N}_{2} \mathrm{O}_{8} \mathrm{Na}_{\text {) }}\right.$ calcd. $581.2833\left([\mathrm{M}+\mathrm{Na}]^{+}\right)$, Found 581.2798. Compound 3 was further characterized by heteronuclear multiple quantum coherence (HMQC) and heteronuclear multiple bond correlation (HMBC).

\subsection{Synthesis of (3R,5S)-Tert-butyl 3-benzyl-5-hydroxyisoxazolidine-2-carboxylate (4a)}

(E)-4-phenylbut-2-enal 1a (146 mg, $1 \mathrm{mmol})$ was dissolved in dichloromethane (5 $\mathrm{mL})$, and then $N$-Boc-hydroxylamine 2 (199 mg, $1.5 \mathrm{mmol})$, (S)-diphenylprolinol-TMS II (33 mg, $0.1 \mathrm{mmol}$ ), and $p$-nitrobenzoic acid (16 mg, $0.1 \mathrm{mmol}, 10 \mathrm{~mol} \%$ ) were added to the resulting solution at $0{ }^{\circ} \mathrm{C}$. The reaction mixture was stirred for $15 \mathrm{~h}$ at $0{ }^{\circ} \mathrm{C}$. The mixture solution was diluted with dichloromethane $(20 \mathrm{~mL})$ and washed with saturated sodium bicarbonate aqueous solution $(5 \mathrm{~mL})$ and brine $(5 \mathrm{~mL})$. The resulting organic phase was dried over $\mathrm{Na}_{2} \mathrm{SO}_{4}$, filtered and concentrated under reduced pressure. The crude product was purified over silica gel column chromatography (PE/EtOAc, 10:1-4:1) to give $4 \mathrm{a}$ as a white solid $(251 \mathrm{mg}, 90 \%)$ mp: $138-139{ }^{\circ} \mathrm{C} ;[\alpha]^{\mathrm{D}}{ }_{22}+16.50\left(\mathrm{c} 1.0, \mathrm{CHCl}_{3}\right)$; ${ }^{1} \mathrm{H}-\mathrm{NMR}\left(400 \mathrm{MHz} \mathrm{CDCl}_{3}\right) \delta 7.29-7.18(\mathrm{~m}, 5 \mathrm{H}), 5.71(\mathrm{~d}, J=4.4 \mathrm{~Hz}, 1 \mathrm{H}), 4.49-4.42(\mathrm{~m}, 1 \mathrm{H}), 3.00$ (dd, $J=8.3 \mathrm{~Hz}, 12 \mathrm{~Hz}, 1 \mathrm{H}), 2.70(\mathrm{dd}, J=8.3 \mathrm{~Hz}, 12 \mathrm{~Hz}, 1 \mathrm{H}), 2.28(\mathrm{dd}, J=8.4 \mathrm{~Hz}, 12 \mathrm{~Hz}, 1 \mathrm{H}), 2.00-1.94(\mathrm{~m}$, 1H), 1.35 (s, 9H) (Supplementary Material Figures S5 and S7); ${ }^{13} \mathrm{C}-\mathrm{NMR}\left(100 \mathrm{MHz}, \mathrm{CDCl}_{3}\right) \delta 158.8$, 138.2, 129.4, 128.3, 126.4, 98.7, 82.0, 59.4, 42.1, 41.3, 28.0 (Supplementary Material Figures S6 and S8); HR-MS $\left(\mathrm{C}_{15} \mathrm{H}_{21} \mathrm{NO}_{4} \mathrm{Na}\right)$ calcd. $302.1363\left([\mathrm{M}+\mathrm{Na}]^{+}\right)$, Found 302.1370; 92\% ee; Chiral HPLC condition: SHIMADZU Essentia LC-16 HPLC, Chiralcel AD-H column $(250 \times 4.6 \mathrm{~mm}$, i.d.) with a mixture of hexane and 2-propanol (95:5) at a flow rate of $1.0 \mathrm{~mL} / \mathrm{min}$ as the mobile phase, oven temperature was $28{ }^{\circ} \mathrm{C}, 210 \mathrm{~nm}$, tminor $=6.66 \mathrm{~min}$, tmajor $=6.00 \mathrm{~min}$. A quintuple scale of this reaction was also carried out and a similar result was observed ( $88 \%$ yield, $92 \%$ ee).

\subsection{Synthesis of (3R)-Tert-butyl 3-benzyl-5-(tert-butoxycarbonyloxy)isoxazolidine-2-carboxylate (5)}

(E)-4-phenylbut-2-enal 1a (146 mg, $1 \mathrm{mmol})$ was dissolved in dichloromethane $(5 \mathrm{~mL})$, and then N-Boc-hydroxylamine 2 (199 mg, $1.5 \mathrm{mmol}$ ), (S)-diphenylprolinol-TMS II (33 mg, $0.1 \mathrm{mmol}$ ), and $p$-Toluenesulfonic acid ( $34 \mathrm{mg}, 0.2 \mathrm{mmol}, 10 \mathrm{~mol} \%$ ) were added to the resulting solution at $0{ }^{\circ} \mathrm{C}$. The reaction mixture was stirred for $8 \mathrm{~h}$ at $0^{\circ} \mathrm{C}$. The mixture solution was diluted with dichloromethane $(20 \mathrm{~mL})$ and washed with saturated sodium bicarbonate aqueous solution $(5 \mathrm{~mL})$ and brine $(5 \mathrm{~mL})$. The resulting organic phase was dried over $\mathrm{Na}_{2} \mathrm{SO}_{4}$, filtered, and concentrated under reduced pressure. The crude product was purified over silica gel column chromatography (PE/EtOAc, 10:1-4:1) to give 5 as a wax solid $(246 \mathrm{mg}, 65 \%) ;[\alpha]^{\mathrm{D}} 22+13.80\left(\right.$ c $\left.1.0, \mathrm{CHCl}_{3}\right) ;{ }^{1} \mathrm{H}-\mathrm{NMR}\left(400 \mathrm{MHz}, \mathrm{CDCl}_{3}\right) \delta 7.30-7.20$ $(\mathrm{m}, 5 \mathrm{H}), 6.11-6.08(\mathrm{~m}, 1 \mathrm{H}), 4.62-4.55(\mathrm{~m}, 1 \mathrm{H}), 2.99(\mathrm{dd}, J=8.6 \mathrm{~Hz}, 12 \mathrm{~Hz}, 1 \mathrm{H}), 2.71(\mathrm{dd}, J=8.5 \mathrm{~Hz}$, $12 \mathrm{~Hz}, 1 \mathrm{H}), 2.63-2.57(\mathrm{~m}, 1 \mathrm{H}), 2.25-2.19(\mathrm{~m}, 1 \mathrm{H}), 1.50(\mathrm{~s}, 9 \mathrm{H}), 1.39$ (s, 9H) (Supplementary Material Figure S11); ${ }^{13} \mathrm{C}-\mathrm{NMR}\left(100 \mathrm{MHz}, \mathrm{CDCl}_{3}\right) \delta$ 158.3, 155.6, 137.7, 129.3, 128.3, 126.4, 88.5, 82.8, 82.2, 61.2, 41.3, 35.2, 28.1, 27.9; HR-MS $\left(\mathrm{C}_{20} \mathrm{H}_{29} \mathrm{NO}_{6} \mathrm{Na}\right)$ calcd. $402.1893\left([\mathrm{M}+\mathrm{Na}]^{+}\right)$, Found 402.1891. 43\% ee; 
Chiral HPLC condition: SHIMADZU Essentia LC-16 HPLC, Chiralcel AD-H column $(250 \times 4.6 \mathrm{~mm}$, i.d.) with a mixture of hexane and 2-propanol (95:5) at a flow rate of $1.0 \mathrm{~mL} / \mathrm{min}$ as the mobile phase, oven temperature was $28{ }^{\circ} \mathrm{C}, 210 \mathrm{~nm}$, tminor $=10.02 \mathrm{~min}$, tmajor $=8.08 \mathrm{~min}$.

\subsection{Synthesis of (3R,5S)-Tert-butyl 5-hydroxy-3-(2,4,5-trifluorobenzyl)isoxazolidine-2-carboxylate (4b)}

(E)-4-(2,4,5-trifluorophenyl)but-2-enal (200 $\mathrm{mg}, 1 \mathrm{mmol})$ was dissolved in dichloromethane (5 mL), and then $N$-Boc-hydroxylamine 2 (159 mg, $1.2 \mathrm{mmol}),(S)$-diphenylprolinol-TMS II (33 mg, 0.1 $\mathrm{mmol}$ ), and p-nitrobenzoic acid (16 $\mathrm{mg}, 0.1 \mathrm{mmol}, 10 \mathrm{~mol} \%$ ) were added to the resulting solution at $-20{ }^{\circ} \mathrm{C}$. The reaction mixture was stirred for $15 \mathrm{~h}$ at $-20^{\circ} \mathrm{C}$. The mixture solution was diluted with dichloromethane $(20 \mathrm{~mL})$ and washed with saturated sodium bicarbonate aqueous solution $(5 \mathrm{~mL})$ and brine $(5 \mathrm{~mL})$. The resulting organic phase was dried over $\mathrm{Na}_{2} \mathrm{SO}_{4}$, filtered and concentrated under reduced pressure. The crude product was purified over silica gel column chromatography (PE/EtOAc, 10:1-4:1) to give $4 \mathbf{b}$ as a white solid $(283 \mathrm{mg}, 85 \%)$. mp: $156-157{ }^{\circ} \mathrm{C} ;[\alpha]^{\mathrm{D}} 22+22.70$ (c 1.0, $\left.\mathrm{CHCl}_{3}\right) ;{ }^{1} \mathrm{H}-\mathrm{NMR}\left(400 \mathrm{MHz} \mathrm{CDCl}_{3}\right) \delta 7.09-7.03(\mathrm{~m}, 1 \mathrm{H}), 6.91-6.85(\mathrm{~m}, 1 \mathrm{H}), 5.70(\mathrm{~d}, J=4.3 \mathrm{~Hz}$, $1 \mathrm{H}), 4.51-4.44(\mathrm{~m}, 1 \mathrm{H}), 2.80(\mathrm{~d}, J=8.8 \mathrm{~Hz}, 2 \mathrm{H}), 2.37(\mathrm{dd}, J=8.7 \mathrm{~Hz}, 12 \mathrm{~Hz}, 1 \mathrm{H}), 1.98-1.92(\mathrm{~m}, 1 \mathrm{H})$, 1.35 (s, 9H) (Supplementary Material Figure S9); ${ }^{13} \mathrm{C}-\mathrm{NMR}\left(100 \mathrm{MHz}, \mathrm{CDCl}_{3}\right) \delta 158.7,156.1,148.8$, 145.1, 121.4, 119.2, 105.1, 98.7, 82.3, 57.9, 41.2, 34.5, 27.9 (Supplementary Material Figure S10); HR-MS $\left(\mathrm{C}_{15} \mathrm{H}_{18} \mathrm{~F}_{3} \mathrm{NO}_{4} \mathrm{Na}\right)$ calcd. $356.1086\left([\mathrm{M}+\mathrm{Na}]^{+}\right)$, Found 356.1087; 96\% ee; Chiral HPLC condition: SHIMADZU Essentia LC-16 HPLC, Chiralcel AD-H column $(250 \times 4.6 \mathrm{~mm}$, i.d.) with a mixture of hexane and 2-propanol (95:5) at a flow rate of $1.0 \mathrm{~mL} / \mathrm{min}$ as the mobile phase, oven temperature was $28^{\circ} \mathrm{C}, 210 \mathrm{~nm}$, tminor $=11.02 \mathrm{~min}$, tmajor $=9.30 \mathrm{~min}$.

\subsection{Synthesis of (R)-N-Boc-3-benzyl-5-oxoisoxazolidine (6a) and (R)-N-Boc-3-trifluorobenzyl-5-oxoisoxazolidine (6b)}

A solution of $\mathrm{NaClO}_{2}(127 \mathrm{mg}, 1.4 \mathrm{mmol})$ in water $(1.4 \mathrm{~mL})$ was added dropwise to a stirred solution of (3R,5S)-tert-butyl-3-benzyl-5-hydroxyisoxazolidine-2-carboxylate $4 \mathrm{a}(279 \mathrm{mg}, 1 \mathrm{mmol}$, $92 \%$ ee) in acetonitrile $(5 \mathrm{~mL})$ and $\mathrm{NaH}_{2} \mathrm{PO}_{4}(24 \mathrm{mg}, 0.2 \mathrm{mmol})$ in water $(1 \mathrm{~mL})$ and $\mathrm{H}_{2} \mathrm{O}_{2}$ (35 W\% in water, $0.14 \mathrm{~mL}, 1.4 \mathrm{mmol}$ ) at $10{ }^{\circ} \mathrm{C}$. The solution was diluted with dichloromethane $(20 \mathrm{~mL})$ and washed with saturated sodium bicarbonate aqueous solution $(5 \mathrm{~mL})$ and brine $(5 \mathrm{~mL})$. The resulting organic phase was dried over $\mathrm{Na}_{2} \mathrm{SO}_{4}$, filtered and concentrated under reduced pressure. The crude product was purified over silica gel column chromatography (PE/EtOAc, 5:1-3:1) to give (R)-N-Boc-3-benzyl-5-oxoisoxazolidine $6 \mathrm{a}\left(227 \mathrm{mg}, 82 \%\right.$ yield) as a white solid. $[\alpha]_{22}^{\mathrm{D}}+40.10$ (c 1.0 , $\left.\mathrm{CHCl}_{3}\right) ;{ }^{1} \mathrm{H}-\mathrm{NMR}\left(400 \mathrm{MHz}, \mathrm{CDCl}_{3}\right) \delta 7.22-7.30(\mathrm{~m}, 5 \mathrm{H}), 4.58-4.65(\mathrm{~m}, 1 \mathrm{H}), 3.04(\mathrm{dd}, J=8.5 \mathrm{~Hz}$, $12 \mathrm{~Hz}, 1 \mathrm{H}), 2.74-2.85(\mathrm{~m}, 2 \mathrm{H}), 2.57(\mathrm{dd}, J=4.7 \mathrm{~Hz}, 16 \mathrm{~Hz}, 1 \mathrm{H}), 1.27(\mathrm{~s}, 9 \mathrm{H})$. Spectroscopic data are in agreement with reference [22]. 92\% ee; Chiral HPLC condition: SHIMADZU Essentia LC-16 HPLC, Chiralcel AD-H column $(250 \times 4.6 \mathrm{~mm}$, i.d. $)$ with a mixture of hexane and 2-propanol (95:5) at a flow rate of $1.2 \mathrm{~mL} / \mathrm{min}$ as the mobile phase, oven temperature was $28^{\circ} \mathrm{C}, 210 \mathrm{~nm}$, tminor $=14.91 \mathrm{~min}$, tmajor $=11.82 \mathrm{~min}$.

Prepared from $4 \mathbf{b}(333 \mathrm{mg}, 1 \mathrm{mmol})$, according to the procedure of the synthesis of $6 \mathbf{a}, \mathbf{6 b}$ was obtained as a white solid (109 mg, 33\%). ${ }^{1} \mathrm{H}-\mathrm{NMR}\left(400 \mathrm{MHz}, \mathrm{CDCl}_{3}\right) \delta 7.29-7.18(\mathrm{~m}, 5 \mathrm{H}), 5.71(\mathrm{~d}$, $J=4.1 \mathrm{~Hz}, 1 \mathrm{H}), 4.49-4.42(\mathrm{~m}, 1 \mathrm{H}), 3.00(\mathrm{dd}, J=8.4 \mathrm{~Hz}, 12 \mathrm{~Hz}, 1 \mathrm{H}), 2.70(\mathrm{dd}, J=8.5 \mathrm{~Hz}, 12 \mathrm{~Hz}, 1 \mathrm{H})$, $2.28(\mathrm{dd}, J=8.4 \mathrm{~Hz}, 12 \mathrm{~Hz}, 1 \mathrm{H}), 2.00-1.94(\mathrm{~m}, 1 \mathrm{H}), 1.35(\mathrm{~s}, 9 \mathrm{H}) ;{ }^{13} \mathrm{C}-\mathrm{NMR}\left(100 \mathrm{MHz}, \mathrm{CDCl}_{3}\right) \delta 173.1$, 158.2, 156.7, 148.1, 146.6, 123.4, 121.9, 107.1, 82.8, 59.2, 42.3, 36.1, 28.9; HR-MS $\left(\mathrm{C}_{15} \mathrm{H}_{16} \mathrm{~F}_{3} \mathrm{NO}_{4} \mathrm{Na}\right)$ calcd. $354.0929\left(\left[\mathrm{M}+\mathrm{Na}^{+}\right)\right.$, Found 354.0927. 96\% ee; Chiral HPLC condition: SHIMADZU Essentia LC-16 HPLC, Chiralcel AD-H column $(250 \times 4.6 \mathrm{~mm}$, i.d. $)$ with a mixture of hexane and 2-propanol (95:5) at a flow rate of $1.2 \mathrm{~mL} / \mathrm{min}$ as the mobile phase, oven temperature was $28^{\circ} \mathrm{C}, 210 \mathrm{~nm}$, tminor $=15.31 \mathrm{~min}$, tmajor $=12.72 \mathrm{~min}$. 
3.9. Synthesis of (R)-N-Boc- $\beta$-benzyl- $\beta$-amino acid 7 a and ( $R)-N$-Boc- $\beta$-(2,4,5-trifluorobenzyl)- $\beta$-amino acid $7 \mathbf{b}$ via oxidation/reductive cleavage of $\mathrm{N}-\mathrm{O}$ bond sequence

To a solution of $(R)-N$-Boc-3-benzyl-5-oxoisoxazolidine 6 a (277 $\mathrm{mg}, 1 \mathrm{mmol}, 92 \%$ ee) in $\mathrm{MeOH}$ $(15 \mathrm{~mL}), \mathrm{Pd} / \mathrm{C}(20 \% \mathrm{w} / w, 55 \mathrm{mg})$ was added. The reaction mixture was hydrogenated under $90 \mathrm{~atm}$ for $24 \mathrm{~h}$. The reaction mixture was filtered through celite with ethyl acetate and concentrated to give (R)-N-Boc- $\beta$-benzyl- $\beta$-amino acid 7a $(276 \mathrm{mg}, 99 \%)$ as a white solid. mp: $111-113{ }^{\circ} \mathrm{C} ;[\alpha]^{\mathrm{D}} 22+18.10$ (c 1.0, $\left.\mathrm{CHCl}_{3}\right) ;{ }^{1} \mathrm{H}-\mathrm{NMR}\left(400 \mathrm{MHz}, \mathrm{CDCl}_{3}\right) \delta 7.33-7.24(\mathrm{~m}, 5 \mathrm{H}), 5.04-4.97(\mathrm{~m}, 1 \mathrm{H}), 2.98(\mathrm{dd}, J=8.4 \mathrm{~Hz}$, 16, 1H), 2.87-2.84 (m, 2H), $2.62(\mathrm{dd}, J=4.3 \mathrm{~Hz}, 16 \mathrm{~Hz}, 1 \mathrm{H}), 1.30(\mathrm{~s}, 9 \mathrm{H})$ (Supplementary Material Figure S12). Spectroscopic data are in agreement with reference [23].

Prepared from $\mathbf{6 b}(331 \mathrm{mg}, 1 \mathrm{mmol})$ according to the procedure of the synthesis of $\mathbf{7 a}, 7 \mathbf{b}$ was obtained as a white solid (330 mg, 99\%). mp: $121-123{ }^{\circ} \mathrm{C} ;[\alpha]^{\mathrm{D}} 22-29.80$ (c 1.0, $\mathrm{CHCl}_{3}$ ) (Supplementary Material Figure S13); ${ }^{1} \mathrm{H}-\mathrm{NMR}\left(400 \mathrm{MHz}, \mathrm{CDCl}_{3}\right) \delta 7.12-7.08(\mathrm{~m}, 1 \mathrm{H}), 6.96-6.90(\mathrm{~m}, 1 \mathrm{H}), 5.09-5.07(\mathrm{~m}, 1 \mathrm{H})$, 4.18-4.15 (m, 1H), 2.92-2.90 (m, 2H), 2.65-2.57 (m, 2H), $1.41(\mathrm{~s}, 9 \mathrm{H})$; Spectroscopic data are in agreement with reference [8].

3.10. Synthesis of (R)-N-Boc- $\beta$-benzyl- $\beta$-amino acid $7 \mathbf{a}$ and (R)-N-Boc- $\beta$-(2,4,5-trifluorobenzyl)- $\beta$-amino acid $7 \mathbf{b}$ via reductive cleavage of $\mathrm{N}-\mathrm{O}$ bond/oxidation sequence

\subsubsection{Method A}

To a solution of (3R,5S)-tert-butyl 3-benzyl-5-hydroxyisoxazolidine-2-carboxylate $4 \mathbf{a}(279 \mathrm{mg}$, $1 \mathrm{mmol}, 92 \%$ ee $)$ in $\mathrm{CH}_{3} \mathrm{CN} / \mathrm{H}_{2} \mathrm{O}\left(6 \mathrm{~mL}, 9: 1\right.$, degassed under nitrogen) was added $\mathrm{Mo}(\mathrm{CO})_{6}(1.2$ equiv., $1.2 \mathrm{mmol})$. The reaction mixture was vigorously stirred and heated to reflux for $4 \mathrm{~h}$. The resulting solution was then filtered through a short column of silica gel (washed by ethyl acetate). The resulting after evaporation was dissolved in dichloromethane $(2 \mathrm{~mL})$ and then it was cooled down to $4{ }^{\circ} \mathrm{C}$. The resulting solution was added isobutene $(1 \mathrm{~mL})$, tert-butanol $(4 \mathrm{~mL}), \mathrm{H}_{2} \mathrm{O}(2 \mathrm{~mL}), \mathrm{KH}_{2} \mathrm{PO}_{4}(544 \mathrm{mg}$, $4 \mathrm{mmol})$, and $\mathrm{NaClO}_{2}(360 \mathrm{mg}, 4 \mathrm{mmol})$, sequentially. The reaction was stirred at this temperature for $12 \mathrm{~h}$. The mixture was diluted with $20 \mathrm{~W} \% \mathrm{NaOH}$ aqueous solution $(10 \mathrm{~mL})$ and dichloromethane $(10 \mathrm{~mL})$. The organic phase was decanted. The aqueous phase was adjusted to $\mathrm{pH}=4-5$ and then extracted with dichloromethane and the combined organic phase was washed with brine, dried over $\mathrm{Na}_{2} \mathrm{SO}_{4}$, and evaporated to give (R)- $N$-Boc- $\beta$-benzyl- $\beta$-amino acid 7a as a white solid (231 $\left.\mathrm{mg}, 83 \%\right)$.

$(R)-N$-Boc- $\beta$-(2,4,5-trifluorobenzyl)- $\beta$-amino acid $7 \mathbf{b}$ was prepared from $4 \mathbf{b}$ (333 $\mathrm{mg}, 1 \mathrm{mmol})$ according to the procedure of the synthesis of $7 \mathbf{a}$, and $7 \mathbf{b}$ was obtained as a white solid $(273 \mathrm{mg}, 82 \%)$.

\subsubsection{Method B}

To a solution of $(3 R, 5 S)$-tert-butyl 3-benzyl-5-hydroxyisoxazolidine-2-carboxylate $4 \mathrm{a}(279 \mathrm{mg}$, $1 \mathrm{mmol}, 92 \%$ ee) in methanol $(10 \mathrm{~mL}), \mathrm{NaBH}_{4}(75.6 \mathrm{mg}, 1 \mathrm{mmol})$ was added carefully at $4{ }^{\circ} \mathrm{C}$, and the reaction mixture was allowed to stir at room temperature for $8 \mathrm{~h}$, and then evaporated. The residue was diluted with water $(20 \mathrm{~mL})$, and then the water phase was extracted with dichloromethane. The combined organic phase was washed with brine, dried over $\mathrm{Na}_{2} \mathrm{SO}_{4}$, and evaporated to give a liquid. The obtained liquid was dissolved in methanol $(20 \mathrm{~mL})$ and the reaction mixture was hydrogenated with Raney Ni (100 mg) at normal pressure for $8 \mathrm{~h}$. The resulting solution was filtered and evaporated. The residue was purified over silica gel column to give (R)-tert-butyl 4-hydroxy-1-phenylbutan-2-ylcarbamate 9a as white solid (220 mg, 83\%). Compound 9a (220 mg, $1.5 \mathrm{mmol}$ ) was dissolved in the mixture solution of dichloromethane (11 mL) and aqueous sodium bicarbonate ( $2.5 \mathrm{~mL}, 5 \% \mathrm{w} / w)$. 2,2,6,6-tetramethylpiperidine-1-oxyl (TEMPO) (13.2 mg, $0.08 \mathrm{mmol})$ and sodium bromide $(8.5 \mathrm{mg}, 0.08 \mathrm{mmol})$ were added to this reaction mixture and it was cooled down to $0{ }^{\circ} \mathrm{C}$. Sodium hypochlorite solution $(3.7 \mathrm{~mL}, 2.5 \mathrm{mmol}, 5 \% \mathrm{w} / \mathrm{w})$ was added dropwise, and then the reaction mixture was stirred for $3 \mathrm{~h}$. The reaction mixture was quenched with saturated aqueous thiosulfate solution $(1 \mathrm{~mL})$, followed by adding aqueous hydrochloric acid (2 M) until the $\mathrm{pH}=4-5$. 
The aqueous phase was extracted with dichloromethane $(10 \mathrm{~mL} \times 3)$. The combined organic phase was dried over $\mathrm{Na}_{2} \mathrm{SO}_{4}$ and concentrated to give compound $7 \mathbf{a}$ as a white solid (206 $\left.\mathrm{mg}, 89 \%\right)$.

$(R)-N$-Boc- $\beta$-(2,4,5-trifluorobenzyl)- $\beta$-amino acid $7 \mathbf{b}$ was prepared from $4 \mathbf{b}$ (333 $\mathrm{mg}, 1 \mathrm{mmol})$ according to the procedure of the synthesis of $7 \mathbf{a}$, and $7 \mathbf{b}$ was obtained as a white solid $(256 \mathrm{mg}, 77 \%$ over two steps).

\subsubsection{Method C}

To a solution of (3R,5S)-tert-butyl 3-benzyl-5-hydroxyisoxazolidine-2-carboxylate $4 \mathbf{a}$ (500 $\mathrm{mg}$, $1.79 \mathrm{mmol}, 92 \%$ ee) in $\mathrm{MeOH}(10 \mathrm{~mL}), \mathrm{Pd} / \mathrm{C}(20 \% w / w, 0.1 \mathrm{~g})$ was added. The reaction mixture was hydrogenated under $90 \mathrm{~atm}$ for $24 \mathrm{~h}$. The reaction mixture was filtered through celite with ethyl acetate and concentrated to give (R)-tert-butyl 4-hydroxy-1-phenylbutan-2-ylcarbamate $9 \mathrm{a}$ as a white solid ( $474 \mathrm{mg}, 99 \%$ ), which $(474 \mathrm{mg}, 1.79 \mathrm{mmol}$ ) was dissolved in the mixture solution of dichloromethane $(24 \mathrm{~mL})$ and aqueous sodium bicarbonate $(5.4 \mathrm{~mL}, 5 \% \mathrm{w} / w)$. TEMPO $(28.8 \mathrm{mg}, 0.18 \mathrm{mmol})$ and sodium bromide $(18.6 \mathrm{mg}, 0.18 \mathrm{mmol})$ were added to this reaction mixture and it was cooled down to $0{ }^{\circ} \mathrm{C}$. Sodium hypochlorite solution $(8 \mathrm{~mL}, 5.4 \mathrm{mmol}, 5 \% \mathrm{w} / \mathrm{w})$ was dropwise added, and then the reaction mixture was stirred for $3 \mathrm{~h}$. The reaction mixture was quenched with saturated aqueous thiosulfate solution $(2 \mathrm{~mL})$, followed by adding aqueous hydrochloric acid $(2 \mathrm{M})$ until the $\mathrm{pH}=4-5$. The aqueous phase was extracted with dichloromethane $(20 \mathrm{~mL} \times 3)$. The combined organic phase was dried over $\mathrm{Na}_{2} \mathrm{SO}_{4}$ and concentrated to give compound $7 \mathbf{a}$ as a white solid (440 $\mathrm{mg}, 89 \%$ ).

(R)- $N$-Boc- $\beta$-(2,4,5-trifluorobenzyl)- $\beta$-amino acid $7 \mathbf{b}$ was prepared from $4 \mathbf{b}$ (333 $\mathrm{mg}, 1 \mathrm{mmol})$ according to the procedure of the synthesis of $\mathbf{7 a}$, and $\mathbf{7 b}$ was obtained as a white solid ( $300 \mathrm{mg}, 90 \%)$.

\subsection{Synthesis of (R)-tert-butyl 4-oxo-1-phenylbutan-2-ylcarbamate (8a) and Synthesis of (R)-tert-butyl 4-oxo-1-2,4,5-trifluorobenzylbutan-2-ylcarbamate (8b)}

To a solution of (3R,5S)-tert-butyl 3-benzyl-5-hydroxyisoxazolidine-2-carboxylate $4 a$ (112 $\mathrm{mg}$, $0.4 \mathrm{mmol}, 92 \%$ ee) in $\mathrm{CH}_{3} \mathrm{CN} / \mathrm{H}_{2} \mathrm{O}\left(4.0 \mathrm{~mL}\right.$, 9:1, degassed under nitrogen), $\mathrm{Mo}(\mathrm{CO})_{6}(126.7 \mathrm{mg}$, $0.48 \mathrm{mmol}, 1.2$ equiv.) was added. The reaction was stirred and heated to reflux for $8 \mathrm{~h}$. The reaction mixture was filtered through a short column of silica gel (washed by EtOAc). The obtained solution was evaporated and purified over silica gel column chromatography (PE/EtOAc, 5:1) to give compound 8a as a white solid. $89 \%$ yield $(93 \mathrm{mg}) ;[\alpha]^{\mathrm{D}}{ }_{22}+18.10\left(\mathrm{c} 1.0, \mathrm{CHCl}_{3}\right) ;{ }^{1} \mathrm{H}-\mathrm{NMR}(400 \mathrm{MHz}, \mathrm{CDCl} 3) \delta 9.69$ $(\mathrm{s}, 1 \mathrm{H}), 7.31-7.21(\mathrm{~m}, 3 \mathrm{H}), 7.15(\mathrm{~d}, J=8.2 \mathrm{~Hz}, 2 \mathrm{H}), 4.75(\mathrm{~s}, 1 \mathrm{H}), 4.25(\mathrm{~s}, 1 \mathrm{H}), 2.95-2.90(\mathrm{~m}, 1 \mathrm{H}), 2.79(\mathrm{dd}$, $J=8.2 \mathrm{~Hz}, 12 \mathrm{~Hz}, 1 \mathrm{H}), 2.63-2.48(\mathrm{~m}, 2 \mathrm{H}), 1.30(\mathrm{~s}, 9 \mathrm{H})[24]$.

(R)-tert-butyl 4-oxo-1-2,4,5-trifluorobenzylbutan-2-ylcarbamate $8 \mathbf{b}$ was prepared from $4 \mathbf{b}$ (333 $\mathrm{mg}$, $1 \mathrm{mmol}$ ) according to the procedure of the synthesis of $\mathbf{8 a}, \mathbf{8 b}$ was obtained as a white solid $(276 \mathbf{m g}$, 87\%): ${ }^{1} \mathrm{H}-\mathrm{NMR}\left(400 \mathrm{MHz}, \mathrm{DMSO}-d_{6}\right) \delta 9.60(\mathrm{~s}, 1 \mathrm{H}), 7.43-7.48(\mathrm{~m}, 1 \mathrm{H}), 7.30-7.32(\mathrm{~m}, 1 \mathrm{H}), 6.88(\mathrm{~d}$, $J=4.7 \mathrm{~Hz}, 1 \mathrm{H}), 4.15-4.17(\mathrm{~m}, 1 \mathrm{H}), 2.55-2.98(\mathrm{~m}, 2 \mathrm{H}), 2.50-2.53(\mathrm{~m}, 2 \mathrm{H}), 1.27(\mathrm{~s}, 9 \mathrm{H})[25]$.

3.12. Synthesis of (R)-Tert-butyl 4-hydroxy-1-phenylbutan-2-ylcarbamate (9a) and (R)-tert-butyl 4-hydroxy-12,4,5-trifluorobenzylbutan-2-ylcarbamate (9b)

To a solution of (3R,5S)-tert-butyl 3-benzyl-5-hydroxyisoxazolidine-2-carboxylate $4 \mathbf{a}$ (500 $\mathrm{mg}$, $1.79 \mathrm{mmol}, 92 \%$ ee) in $\mathrm{MeOH}(10 \mathrm{~mL}), \mathrm{Pd} / \mathrm{C}(20 \% w / w, 0.1 \mathrm{~g})$ was added. The reaction mixture was hydrogenated under $90 \mathrm{~atm}$ for $24 \mathrm{~h}$. The reaction mixture was filtered through celite with ethyl acetate and concentrated to give compound $9 \mathrm{a}$ as a white solid. $99 \%$ yield $(474 \mathrm{mg}) ;[\alpha]^{\mathrm{D}} 22-2.50$ (c 1.0, $\left.\mathrm{CHCl}_{3}\right) ;{ }^{1} \mathrm{H}-\mathrm{NMR}\left(400 \mathrm{MHz}, \mathrm{CDCl}_{3}\right) \delta 7.34-7.20(\mathrm{~m}, 5 \mathrm{H}), 4.49(\mathrm{~d}, J=12.1 \mathrm{~Hz}, 1 \mathrm{H}), 4.15-4.07(\mathrm{~m}$, $1 \mathrm{H}), 3.67(\mathrm{~s}, 2 \mathrm{H}), 3.18-3.12(\mathrm{~m}, 1 \mathrm{H}), 2.83(\mathrm{~d}, J=4.7 \mathrm{~Hz}, 2 \mathrm{H}), 1.91-1.83(\mathrm{~m}, 1 \mathrm{H}), 1.43(\mathrm{~s}, 9 \mathrm{H}) ;{ }^{13} \mathrm{C}-\mathrm{NMR}$ $\left(100 \mathrm{MHz}, \mathrm{CDCl}_{3}\right) \delta 156.8,138.8,129.3,128.2,126.2,81.0,59.6,58.2,38.4,33.9,28.1$ [26].

(R)-tert-butyl 4-hydroxy-1-2,4,5-trifluorobenzylbutan-2-ylcarbamate $\mathbf{9 b}$ was prepared from $\mathbf{4 b}$ (333 $\mathrm{mg}, 1 \mathrm{mmol}$ ) according to the procedure of the synthesis of $\mathbf{9 a}$, and $\mathbf{9} \mathbf{b}$ was obtained as a white solid (316 mg, 99\%): ${ }^{1} \mathrm{H}-\mathrm{NMR}\left(400 \mathrm{MHz}, \mathrm{CDCl}_{3}\right)$ 8 7.03-7.10 (m, 1H), 6.90-6.96 (m, 1H), 4.49 (d, J = $12.5 \mathrm{~Hz}$, 
$1 \mathrm{H}), 4.57(\mathrm{~d}, J=12.6 \mathrm{~Hz}, 1 \mathrm{H}), 4.00-4.06(\mathrm{~m}, 1 \mathrm{H}), 3.69(\mathrm{~d}, J=4.2 \mathrm{~Hz}, 1 \mathrm{H}), 2.75-2.82(\mathrm{~m}, 2 \mathrm{H}), 1.84-1.92(\mathrm{~m}$, $2 \mathrm{H}), 1.42(\mathrm{~s}, 9 \mathrm{H})[13]$.

\subsection{Synthesis of Sitagliptin Phosphate Monohydrate}

To a solution of $7 \mathbf{b}(1 \mathrm{~g}, 3.0 \mathrm{mmol})$ in DCM $(10 \mathrm{~mL})$, EDCI (0.69 g, $3.6 \mathrm{mmol})$, HOBT (0.49 g, $3.6 \mathrm{mmol}), 3$-(trifluoromethyl)-5,6,7,8-tetrahydro-1,2,4-triazolo[4,3-a]pyrazine 10 (0.58 g, $3.0 \mathrm{mmol})$, and DIPEA ( $0.39 \mathrm{~g}, 3.0 \mathrm{mmol})$ were added successively. The reaction mixture was stirred for $12 \mathrm{~h}$ at room temperature and then $\mathrm{H}_{2} \mathrm{O}(10 \mathrm{~mL})$ and ethyl acetate $(20 \mathrm{~mL})$ were added to the reaction mixture. The organic phase was washed with saturated aqueous $\mathrm{NaHCO}_{3}$ solution $(5 \mathrm{~mL})$, aqueous $\mathrm{HCl}$ solution $(5 \mathrm{~mL}, 1 \mathrm{~N})$, brine $(5 \mathrm{~mL})$, and dried over with anhydrous $\mathrm{Na}_{2} \mathrm{SO}_{4}$. The solvent was evaporated and the residue was purified over silica gel chromatography to afford $\mathbf{1 1}$ as a foamy solid (1.38 g, 91\%). Compound 11 (1.38 g, $2.7 \mathrm{mmol})$ was dissolved in $60 \mathrm{~mL}$ of saturated methanolic hydrogen chloride solution and stirred for $1 \mathrm{~h}$. The solution was concentrated and partitioned between ethyl acetate $(40 \mathrm{~mL})$ and $1 \mathrm{~N}$ aqueous sodium hydroxide solution $(40 \mathrm{~mL})$. The aqueous layer was extracted with ethyl acetate $(3 \times 40 \mathrm{~mL})$. The combined organic phase was evaporated and immediately dissolved in isopropanol $(4 \mathrm{~mL})$. The solution was maintained at $40^{\circ} \mathrm{C}$ for $1 \mathrm{~h}$ and then cooled down to $20-15^{\circ} \mathrm{C}$. Heptane $(12 \mathrm{~mL})$ was added dropwise over $30 \mathrm{~min}$ and filtered. The wet cake was washed with $20 \%$ isopropanol in heptanes and dried over vacuo to give free base of $11(0.89 \mathrm{~g}, 81 \%,>99.9 \%$ ee $)$. The obtained solid $(0.89 \mathrm{~g}, 2.2 \mathrm{mmol})$ was dissolved in isopropanol $(3.8 \mathrm{~mL})$ and $\mathrm{H}_{2} \mathrm{O}(1 \mathrm{~mL})$ and $45 \%$ $\mathrm{H}_{3} \mathrm{PO}_{4}(2.5 \mathrm{mmol})$ were added dropwise. The mixture was heated to $70-80{ }^{\circ} \mathrm{C}$ to dissolve the solids and then cooled to $60-65^{\circ} \mathrm{C}$ and seeded with trace sitagliptin phosphate monohydrate. The batch was aged for $1 \mathrm{~h}$ and cooled to ambient temperature. 2-Propanol $(2.8 \mathrm{~mL})$ was added to the batch slowly and then filtered. The wet cake was washed with aqueous isopropanol ( $20 \%$ water, $3 \mathrm{~mL}$ ), and dried over vacuo at $60{ }^{\circ} \mathrm{C}$ to give sitagliptin phosphate monohydrate $\mathbf{1}(1.1 \mathrm{~g}, 96 \%$ yield, $>99.9 \%$ ee). Chiral HPLC condition: SHIMADZU Essentia LC-16 HPLC, Chiralpak IC-3 column with a mixture of $40 \%$ hexanes ( $0.1 \%$ diethylamine), $60 \%$ 2-PrOH ( $0.1 \%$ diethylamine) at a flow rate of $0.5 \mathrm{~mL} / \mathrm{min}$ as the mobile phase, oven temperature was $28{ }^{\circ} \mathrm{C}, 210 \mathrm{~nm}$, tminor $=21.12 \mathrm{~min}$, tmajor $=19.10 \mathrm{~min}$. Data of sample was consistent with that of standard sample [6].

\section{Conclusions}

In summary, we disclosed an efficient and practical route for the asymmetric synthesis of sitagliptin phosphate monohydrate using chiral hemiactal as the key intermediate starting from (E)-4-(2,4,5-trifluorophenyl)but-2-enal. The chiral hemiacetal fragment was constructed by tandem aza-Michael/hemiacetal reaction catalyzed with organocatalyst, without the use of expensive metal catalyst. Furthermore, the influence of acidity of Brønsted acid on tandem aza-Michael/hemiacetal reaction was addressed in detail.

Supplementary Materials: Supplementary Materials are available online.

Author Contributions: Methodology: H.G., J.Y., C.G., Q.J.; Formal Analysis, H.G., C.G.; Investigation, H.G., J.Y., C.G., Q.J.; Writing-Original Draft Preparation, C.G.; Writing-Review \& Editing, J.Y.

Funding: This research was funded by National Natural Science Foundation of China (No. 21472110), Zhejiang Provincial National Science Foundation of China (LQ18B020003) and Zhejiang Qianjiang Talent Program.

Conflicts of Interest: The authors declare no conflicts of interest.

\section{References}

1. Thornberry, N.A.; Weber, A.E. Discovery of JANUVIA ${ }^{\mathrm{Tm}}$ (Sitagliptin), a Selective Dipeptidyl Peptidase IV Inhibitor for the Treatment of Type2 Diabetes. Curr. Top. Med. Chem. 2007, 7, 557-568. [CrossRef] [PubMed]

2. Shultz, C.S.; Krska, S.W. Unlocking the potential of asymmetric hydrogenation at Merck. Acc. Chem. Res. 2007, 40, 1320-1326. [CrossRef] [PubMed] 
3. Xia, L.-H. Sitagliptin: A new tihyperglycemic agent. Chin. J. New Drug 2007, 16, 979-981.

4. Desai, A.A. Sitagliptin Manufacture: A Compelling Tale of Green Chemistry, Process Intensification, and Industrial Asymmetric Catalysis. Angew. Chem. Int. Ed. 2011, 50, 1974-1976. [CrossRef] [PubMed]

5. Savile, C.K.; Janey, J.M.; Mundorff, E.C.; Moore, J.C.; Tam, S.; Jarvis, W.R.; Colbeck, J.C.; Krebber, A.; Fleitz, F.J.; Brands, J.; et al. Biocatalytic asymmetric synthesis of chiral amines from ketones applied to Sitagliptin manfacture. Science 2010, 329, 305-309. [CrossRef] [PubMed]

6. Hansen, K.B.; Hsiao, Y.; Xu, F.; Rivera, N.; Clausen, A.; Kubryk, M.; Krska, S.; Rosner, T.; Simmons, B.; Balsells, J.; et al. Highly efficient asymmetric synthesis of sitagliptin. J. Am. Chem. Soc. 2009, 131, 8798-8804. [CrossRef] [PubMed]

7. Steinhuebel, D.; Sun, Y.; Matsumura, K.; Sayo, N.; Saito, T. Direct asymmetric reductive amination. J. Am. Chem. Soc. 2009, 131, 11316-11317. [CrossRef] [PubMed]

8. Zhou, S.; Wang, J.; Chen, X.; Aceña, J.L.; Soloshonok, V.A.; Liu, H. Chemical kinetic resolution of unprotected $\beta$-substituted $\beta$-amino acids using recyclable chiral ligands. Angew. Chem. Int. Ed. 2014, 53, 7883-7886. [CrossRef] [PubMed]

9. Reddy, P.P.; Babu, I.; Srinivas, P.; Shailaja, P.; Kavitha, N.; Anand, R.V.; Reddy, V.R. Process for the Preparation of Sitaliptin and Pharmaceutically Acceptable Salts Thereof. U.S. Patent 8,969,558, 3 March 2015.

10. Lin, K.; Cai, Z.; Zhou, W. Practical and economical approach to synthesize sitagliptin. Synth. Commun. 2013, 43, 3281-3286. [CrossRef]

11. Davies, S.G.; Fletcher, A.M.; Lv, L.; Roberts, P.M.; Thomson, J.E. Asymmetric synthesis of (-)-(R)-sitagliptin. Tetrahedron Lett. 2012, 53, 3052-3055. [CrossRef]

12. Subbaiah, C.S.; Haq, W. Efficient stereocontrolled synthesis of sitagliptin phosphate. Tetrahedron Asymmetry 2014, 25, 1026-1030. [CrossRef]

13. Pan, X.; Li, X.; Lu, Q.; Yu, W.; Li, W.; Zhang, Q.; Deng, F.; Liu, F. Efficient synthesis of sitagliptin phosphate, a novel DPP-IV inhibitor via a chiral aziridine intermediate. Tetrahedron Lett. 2013, 54, 6807-6809. [CrossRef]

14. Pan, X.; Bai, S.; Yu, W.; Ding, D.; Zhao, D.; Liu, F. Efficient synthesis of 3-R-Boc-amino-4-(2,4,5-trifluorophenyl) butyric Acid. Synth. Commun. 2015, 45, 1451-1456. [CrossRef]

15. Jiang, H.; Gao, H.; Ge, C. Concise synthesis of valuable chiral $N$-Boc- $\beta$-benzyl- $\beta$-amino acid via construction of chiral N-Boc-3-benzyl-5-oxoisoxazolidine through cross-Metathesis/conjugate addition/oxidation. Chin. Chem. Lett. 2017, 28, 471-475. [CrossRef]

16. Chen, Y.K.; Yoshida, M.; MacMillan, D.W.C. Enantioselective organocatalytic amine conjugate addition. J. Am. Chem. Soc. 2006, 128, 9328-9329. [CrossRef] [PubMed]

17. Ibrahem, I.; Rios, R.; Vesely, J.; Zhao, G.-L.; Córdova, A. Organocatalytic asymmetric 5-hydroxyisoxazolidine synthesis: A highly enantioselective route to $\beta$-amino acids. Chem. Commun. 2007, 849-851. [CrossRef] [PubMed]

18. Maltsev, O.V.; Kucherenko, A.S.; Chimishkyan, A.L.; Zlotin, S.G. $\alpha, \alpha$-Diarylprolinol-derived chiral ionic liquids: Recoverable organocatalysts for the domino reaction between $\alpha, \beta$-enals and $N$-protected hydroxylamines. Tetrahedron Asymmetry 2010, 21, 2659-2670. [CrossRef]

19. Zhao, G.-L.; Lin, S.; Korotvička, A.; Deiana, L.; Kullberg, M.; Córdova, A. Asymmetric Synthesis of Maraviroc (UK-427,857). Adv. Synth. Catal. 2010, 352, 2291-2298. [CrossRef]

20. Gao, H. pKa values was measured in water. In $p K a$ Predictions for Organic Acids and Bases; Perrin, D.D., Serjeant, E.P., Dempsey, B., Eds.; Champman and Hall: London, UK, 1981.

21. Albrecht, Ł.; Dickmeiss, G.; Acosta, F.C.; Rodriguez-Escrich, C.; Davis, R.L.; Jørgensen, K.A. Asymmetric Organocatalytic Formal [2 + 2]-Cycloadditions via Bifunctional H-Bond Directing Dienamine Catalysis. J. Am. Chem. Soc. 2012, 134, 2543-2546. [CrossRef] [PubMed]

22. Juarez-Garcia, M.E.; Yu, S.; Bode, J.W. Asymmetric synthesis of enantiopure isoxazolidinone monomers for the synthesis of $\beta^{3}$-oligopeptides by chemoselective amide ligation. Tetrahedron 2010, 66, 4841-4853. [CrossRef] [PubMed]

23. Seki, M.; Matsumoto, K. A novel approach to homochiral $\beta$-amino acids. Tetrahedron Lett. 1996, 37, 3165-3168. [CrossRef]

24. Chen, H.G.; Tustin, J.M.; Wuts, P.G.M.; Sawyer, T.K.; Smith, C.W. Stereoselective synthesis of Xaay $\left[\mathrm{CH}_{2} \mathrm{CH}(\mathrm{OH})\right]$ Yaa dipeptidomimetics and their inclusion in HIV-1 protease inhibitoes. Int. J. Pept. Protein Res. 1995, 45, 1-10. [CrossRef] [PubMed]

25. Kaspar, Z.; Kaspar, B.D.; Saliha, M. Compounds of Formula (I) as Serine Protease Inhibitors. PCT Patent: WO2008101953, 28 August 2008. 
26. Machida, S.; Usuba, K.; Blaskovich, M.A.; Yano, A.; Harada, K.; Sebti, S.M.; Kato, N.; Ohkanda, J. Module Assembly for Protein-Surface Recognition: Geranylgeranyltransferase I Bivalent Inhibitors for Simultaneous Targeting of Interior and Exterior Protein Surfaces. Chem. Eur. J. 2008, 14, 1392-1401. [CrossRef] [PubMed]

Sample Availability: Samples of the compounds (1a, 1b, 2-11 and sitagliptin phosphate monohydrate) are available from the authors.

(C) 2018 by the authors. Licensee MDPI, Basel, Switzerland. This article is an open access article distributed under the terms and conditions of the Creative Commons Attribution (CC BY) license (http://creativecommons.org/licenses/by/4.0/). 